\title{
Numerically Stable Evaluation of Moments of Random Gram Matrices with Applications
}

\author{
Khalil Elkhalil, Student Member, IEEE, Abla Kammoun, Member, IEEE, Tareq Y. Al-Naffouri, Member, IEEE, \\ and Mohamed-Slim Alouini, Fellow, IEEE
}

\begin{abstract}
This paper focuses on the computation of the positive moments of one-side correlated random Gram matrices. Closed-form expressions for the moments can be obtained easily, but numerical evaluation thereof is prone to numerical stability, especially in high-dimensional settings. This letter provides a numerically stable method that efficiently computes the positive moments in closed-form. The developed expressions are more accurate and can lead to higher accuracy levels when fed to moment based-approaches. As an application, we show how the obtained moments can be used to approximate the marginal distribution of the eigenvalues of random Gram matrices.
\end{abstract}

Index Terms-Gram matrices, one sided correlation, positive moments, Laguerre polynomials.

\section{INTRODUCTION}

G RAM random matrices with one-sided correlation naturally arise in the context of signal processing [1] and wireless communications [2]3]. For instance, in signal processing, inverse moments of this kind of matrices are used to evaluate the performance of linear estimators such as the best linear unbiased estimator (BLUE) and optimize the design of some covariance matrix estimators [14]]. In wireless communications, Gram random matrices arise as a key element in the computation of the ergodic capacity of amplify and forward (AF) multiple input multiple output (MIMO) dualhop systems [3].

Given a random matrix $\mathbf{H} \in \mathbb{C}^{q \times n_{t}}$ such that $\mathbf{H}=\boldsymbol{\Lambda}^{\frac{1}{2}} \mathbf{X}$, where $\mathbf{X}$ is a standard complex Gaussian random matrix and $\boldsymbol{\Lambda}$ is a Hermitian positive definite matrix, the authors in [3] derive a closed form expression of the marginal probability density function (PDF) of an unordered eigenvalue of the Gram matrix $\mathbf{W}=\mathbf{H}^{H} \mathbf{H}$ for arbitrary dimensions $n_{t}$ and $q$. The result, although being very useful, involves the inversion of a large Vandermonde matrix and as such might not be always numerically stable, especially in the following situations:

- The dimensions $q$ belongs to moderate to large values.

- The gap between the eigenvalues of $\boldsymbol{\Lambda}$ is small.

To solve this problem, an expression of the exact marginal PDF has been proposed in [5] when some eigenvalues are identical. However, the problem of numerical stability remains when some eigenvalues of $\boldsymbol{\Lambda}$ are different but close to each other. Motivated by these facts, we provide a more stable method to compute the positive moments of $\mathbf{W}$ without the need to

K. Elkhalil, A. Kammoun, T. Y. Al-Naffouri and M.-S. Alouini are with the Electrical Engineering Program, King Abdullah University of Science and Technology, Thuwal, Saudi Arabia; e-mails: \{khalil.elkhalil, abla.kammoun, tareq.alnaffouri, slim.alouini $\} @$ kaust.edu.sa. This work was funded by a CRG3 grant from the office of competitive research (OCRF) at KAUST. invert large Vandermonde matrices. The contributions of this letter are summarized as follows:

- We provide a numerically stable method to evaluate the positive moments of $\mathbf{W}$.

- Using Laguerre polynomials and based on the calculated positive moments, we provide a numerically stable approximation of the marginal probability density function (PDF) of the eigenvalues of $\mathbf{W}$.

The remainder of this letter is organized as follows. In Section $[$ II. we provide the main steps to efficiently compute the positive moments of $\mathbf{W}$. In Section III] we propose to approximate the marginal PDF based on the computed positive moments using Laguerre polynomials. In Section [V], we present some numerical results to validate our method and finally we conclude our work in Section $\mathrm{V}$.

\section{A NumericAlly STABle METHOd to COMPUTE THE MOMENTS OF RANDOM GRAM MATRICES}

\section{A. Problem statement}

Let $\mathbf{H}=\boldsymbol{\Lambda}^{\frac{1}{2}} \mathbf{X} \in \mathbb{C}^{q \times n_{t}}$ where $\boldsymbol{\Lambda} \in \mathbb{C}^{q \times q}$ is a positive definite matrix with distinct eigenvalues $0<\beta_{1}<\beta_{2}<\cdots<$ $\beta_{q}$ and $\mathbf{X}$ a standard complex Gaussian matrix. Assume that $n_{t} \leq q$. The marginal PDF of an unordered eigenvalue $\lambda$ of $\mathbf{W}=\mathbf{H}^{H} \mathbf{H}$ is given by [3, Lemma 1]

$$
\begin{aligned}
f_{\lambda}(\lambda) & =\frac{1}{n_{t} \prod_{i<j}^{q}\left(\beta_{j}-\beta_{i}\right)} \\
& \times \sum_{l=1}^{q} \sum_{k=q-n_{t}+1}^{q} \frac{\lambda^{n_{t}+k-q-1} e^{-\lambda / \beta_{l}} \beta_{l}^{q-n_{t}-1}}{\Gamma\left(n_{t}-q+k\right)} D_{l, k},
\end{aligned}
$$

where $D_{l, k}=\{\mathbf{D}\}_{l, k}$ is the $(l, k)$ th cofactor of the Vandermonde $q \times q$ matrix $\Psi$ whose $(m, n)$ th entry is

$$
\{\boldsymbol{\Psi}\}_{m, n}=\beta_{m}^{n-1} .
$$

Expressing the inverse of $\Psi$ as

$$
\boldsymbol{\Psi}^{-1}=\frac{1}{\operatorname{det}(\boldsymbol{\Psi})} \mathbf{D}^{T}=\frac{1}{\prod_{i<j}^{q}\left(\beta_{j}-\beta_{i}\right)} \mathbf{D}^{T},
$$

the PDF in (1) simplifies to

$$
f_{\lambda}(\lambda)=\frac{1}{n_{t}} \sum_{l=1}^{q} \sum_{k=q-n_{t}+1}^{q} \frac{\lambda^{n_{t}+k-q-1} e^{-\lambda / \beta_{l}} \beta_{l}^{q-n_{t}-1}}{\Gamma\left(n_{t}-q+k\right)} \Psi_{k, l}^{-1}
$$


The cumulative density function (CDF) can thus be easily derived as

$$
F_{\lambda}(\lambda)=\frac{1}{n_{t}} \sum_{l=1}^{q} \sum_{k=q-n_{t}+1}^{q} \frac{\beta_{l}^{k-1} \gamma\left(n_{t}-q+k, \lambda / \beta_{l}\right)}{\Gamma\left(n_{t}-q+k\right)} \Psi_{k, l}^{-1},
$$

where $\Gamma($.$) and \gamma(.,$.$) are respectively the standard Gamma$ and the lower incomplete Gamma functions.

Knowing the marginal PDF, it is possible to compute the expected value of any functional $g$ of the eigenvalues of $\mathbf{W}$. Indeed, we have

$$
\mathbb{E}[g(\mathbf{W})] \triangleq \frac{1}{n_{t}} \sum_{i=1}^{n_{t}} g\left(\lambda_{i}(\mathbf{W})\right)=\int_{0}^{\infty} g(\lambda) f_{\lambda}(\lambda) d \lambda,
$$

where $\left\{\lambda_{i}(\mathbf{W})\right\}_{i=1}^{n_{t}}$ are the eigenvalues of $\mathbf{W}$. The above equation is very useful in practice as it can be leveraged to compute performance metrics of many wireless communication and signal processing schemes. Examples include the ergodic capacity, the SINR at the output of the MMSE receiver and the MSE of the BLUE estimator which correspond respectively to selecting $g(x)$ as $g(x)=\log _{2}\left(x+\sigma^{2}\right), g(x)=\frac{1}{x+\sigma^{2}}$ where $\sigma^{2}$ is the noise variance and $g(x)=x^{-1}$.

When it comes to numerically compute $\mathbb{E}[g(\mathbf{W})]$, it is easy to see that, when the eigenvalues $\left\{\beta_{i}\right\}$ are very close causing the matrix $\Psi$ to be ill-conditioned, some numerical stability issues will occur. In this work, we show that for some functionals $g$, namely polynomials, it is possible to evaluate $\mathbb{E}[g(\mathbf{W})]$ in a stable way. This allows us, using moment approximation techniques, to obtain a numerically stable approximation of the marginal PDF. We believe that the same approximation method can also be extended to approximate $\mathbb{E}[g(\mathbf{W})]$ for any functional $g$ of interest.

\section{B. A Numerically stable method to compute positive moments}

In this section, we propose a numerically stable technique to compute the positive moments of $\mathbf{W}$. Let $p \in \mathbb{N}$, the $p$-th moment of matrix $\mathbf{W}$ is given by

$$
\begin{aligned}
\mu_{\mathbf{W}}(p) & \triangleq \mathbb{E}\left[\lambda^{p}\right]=\frac{1}{n_{t}} \mathbb{E} \operatorname{tr}\left[\mathbf{W}^{p}\right]=\int_{0}^{\infty} \lambda^{p} f_{\lambda}(\lambda) \\
& =\frac{1}{n_{t}} \sum_{k=q-n_{t}+1}^{q} \frac{\Gamma\left(n_{t}+p+k-q\right)}{\Gamma\left(n_{t}-q+k\right)} \sum_{l=1}^{q} \mathbf{\Psi}_{k, l}^{-1} \beta_{l}^{p+k-1} .
\end{aligned}
$$

In many practical scenarios, numerical instability originates from the computation of the following quantity

$$
\sum_{l=1}^{q} \mathbf{\Psi}_{k, l}^{-1} \beta_{l}^{p+k-1}
$$

as $\boldsymbol{\Psi}$ is ill-conditioned. To overcome this issue, we propose an alternative way that avoids computing the inverse of $\boldsymbol{\Psi}$. For $k \in \llbracket 1, q \rrbracket$ and $\tau \in \llbracket p+q-n_{t}, p+q-1 \rrbracket$, define $\alpha_{k, \tau}$ as

$$
\alpha_{k, \tau}=\sum_{l=1}^{q} \boldsymbol{\Psi}_{k, l}^{-1} \beta_{l}^{\tau}
$$

The basic idea is based on the observation that $\boldsymbol{\alpha}_{\tau} \triangleq$ $\left[\alpha_{1, \tau}, \cdots, \alpha_{q, \tau}\right]^{T}$ is solution to the following linear system

$$
\boldsymbol{\Psi} \boldsymbol{\alpha}_{\tau}=\boldsymbol{\beta}_{\tau}
$$

where $\boldsymbol{\beta}_{\tau}=\left[\beta_{1}^{\tau}, \cdots, \beta_{q}^{\tau}\right]^{T}$. If $0 \leq \tau \leq q$, then a straightforward solution to $(6)$ is given by $\boldsymbol{\alpha}_{\tau}=\left[\mathbf{0}_{\tau \times 1}^{T}, 1, \mathbf{0}_{q-\tau \times 1}^{T}\right]^{T}$. From now on, we assume that $\tau>q$.

Writing (6) in the following equivalent way

$$
\beta_{k}^{\tau}=\sum_{l=1}^{q} \beta_{k}^{l} \alpha_{l, \tau}, k=1, \cdots, q
$$

we can easily see that $\left\{\beta_{i}\right\}_{i=1}^{q}$ are roots of the following polynomial:

$$
P(X)=\sum_{k=1}^{q} \alpha_{k, \tau} X^{k-1}-X^{\tau} .
$$

Hence, there exists $Q(X)$ a polynomial with degree $\tau-q$ such that:

$$
P(X)=Q(X) \prod_{i=1}^{q}\left(X-\beta_{i}\right),
$$

Note that exact knowledge of $P(X)$ leads to the determination of the unknown coefficients $\alpha_{k, \tau}$, since they are by construction among the coefficients of $P(X)$. To fully characterize $P(X)$, we first observe that

- the coefficients of $P$ associated with exponents $X^{q}, X^{q+1}, \cdots, X^{\tau-1}$ are all zero.

- the coefficient associated with $X^{\tau}=-1$.

Let $\left\{a_{i}\right\}_{i=1}^{q+1}$ be the coefficients of $\prod_{i=1}^{q}\left(X-\beta_{i}\right)$ (i.e, $\left.\prod_{i=1}^{q}\left(X-\beta_{i}\right)=\sum_{i=1}^{q+1} a_{i} X^{i-1}\right)$, which can be exactly obtained using the Newton-Girard algorithm [6]. Let $\left\{b_{i}\right\}_{i=1}^{\tau-q+1}$ be the coefficients of $Q(X)$ so that: $Q(X)=$ $\sum_{k=1}^{\tau-q} b_{k} X^{k-1}$. From the available information about the coefficients of $P(X)$, we can show that $\left\{b_{k}\right\}_{k=1}^{\tau-q+1}$ satisfy

$$
\boldsymbol{\Phi}\left[\begin{array}{c}
b_{1} \\
b_{2} \\
\vdots \\
b_{\tau-q+1}
\end{array}\right]=\left[\begin{array}{c}
0 \\
0 \\
\vdots \\
0 \\
-1
\end{array}\right],
$$

where $\boldsymbol{\Phi}$ is the upper triangular matrix given by

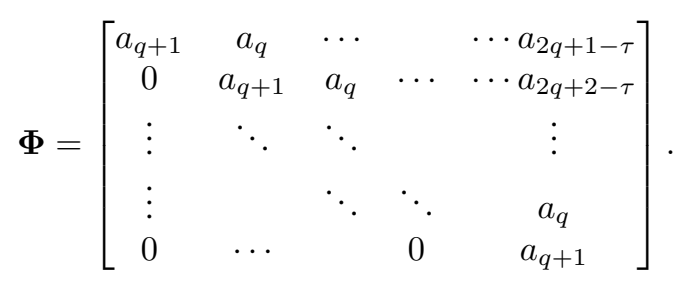

The vector $\mathbf{b}=\left[b_{1}, \cdots, b_{\tau-q+1}\right]^{T}$ can thus be determined by taking the inverse of matrix $\boldsymbol{\Phi}$ as $\mathbf{b}=\boldsymbol{\Phi}$ । $[z \operatorname{eros}(1, \tau-q),-1]^{T}$. From a numerical standpoint, this operation, involving inversion of an upper triangular matrix, can be solved in a stable fashion using back-substitution algorithm and is, as such, much more stable than the inversion of matrix $\boldsymbol{\Psi}$ required in the evaluation of (5). Once 
Table I: Positive moments evaluation of the Gram matrix W with $\Lambda$ as in (11) with both $\xi=0.85$ and $\xi=0.95$. Both configurations are considered: $c_{1}: n_{t}=3, q=5$ and $c_{2}$ : $n_{t}=3, q=20$ for different moment order values, $p$.

\begin{tabular}{|c|c|c|c|c|c|}
\hline & & Exact [3] & Empirical & Proposed & Rel. Error \\
\hline \multirow{2}{*}{$c_{1}(p=1)$} & $\xi=0.85$ & 0.5563 & 0.5564 & 0.5563 & $1.111 \mathrm{e}-04$ \\
\hline & $\xi=0.95$ & 0.2262 & 0.2262 & 0.2262 & $4.9983 \mathrm{e}-05$ \\
\hline \multirow{2}{*}{$c_{2}(p=1)$} & $\xi=0.85$ & $5.5219 \mathrm{e}-11$ & 0.9612 & 0.9612 & $8.5393 \mathrm{e}-05$ \\
\hline & $\xi=0.95$ & $6.6397 \mathrm{e}-26$ & 0.6415 & 0.6415 & $7.3081 \mathrm{e}-06$ \\
\hline \multirow{2}{*}{$c_{1}(p=5)$} & $\xi=0.85$ & 1.1033 & 1.1035 & 1.1033 & $2.2597 \mathrm{e}-04$ \\
\hline & $\xi=0.95$ & 0.0110 & 0.0110 & 0.0110 & $1.4768 \mathrm{e}-04$ \\
\hline \multirow{2}{*}{$c_{2}(p=5)$} & $\xi=0.85$ & $5.7674 \mathrm{e}-12$ & 4.7575 & 4.7563 & $2.6387 \mathrm{e}-04$ \\
\hline & $\xi=0.95$ & $7.9630 \mathrm{e}-29$ & 0.3587 & 0.3586 & $1.8209 \mathrm{e}-04$ \\
\hline \multirow{2}{*}{$c_{1}(p=8)$} & $\xi=0.85$ & 5.3989 & 5.3940 & 5.3989 & $9.1688 \mathrm{e}-04$ \\
\hline & $\xi=0.95$ & 0.0031 & 0.0031 & 0.0031 & $9.3266 \mathrm{e}-04$ \\
\hline \multirow{2}{*}{$c_{2}(p=8)$} & $\xi=0.85$ & $8.3850 \mathrm{e}-12$ & 37.4839 & 37.4747 & $2.4517 \mathrm{e}-04$ \\
\hline & $\xi=0.95$ & $4.2675 \mathrm{e}-30$ & 0.4387 & 0.4386 & $2.9514 \mathrm{e}-04$ \\
\hline
\end{tabular}

coefficients $\left\{b_{i}\right\}_{i=1}^{\tau-q+1}$ are obtained, $\left\{\alpha_{k, \tau}\right\}$ can be evaluated as $11 \alpha_{j, \tau}=\sum_{k=1}^{\tau-q+1} b_{k} a_{j+2-k}$. To validate our procedure, we compute the positive moments of the Gram matrix $\mathbf{W}$ in the case where the correlation matrix $\boldsymbol{\Lambda}$ follows the following model [1]

$$
\boldsymbol{\Lambda}=(1-\xi) \operatorname{diag}\left(1, \xi, \xi^{2}, \cdots, \xi^{q-1}\right), 0 \leq \xi \leq 1,
$$

where the coefficient $\xi$ indicates the forgetting factor. This kind of matrices arise in covariance matrix estimation and more precisely in exponentially weighted sample covariance matrix (more details can be found in [1], section III-B) . Note that for moderate to large values of $q$, the eigenvalues of $\boldsymbol{\Lambda}$ given by $(1-\xi),(1-\xi) \xi, \cdots,(1-\xi) \xi^{q-1}$ are very close to each other, which promotes singularity issues when using the formula in (1). We consider two different configurations $c_{1}$ and $c_{2}$ corresponding respectively to $\left(n_{t}=3, q=5\right)$ and $\left(n_{t}=3, q=20\right)$. For both configurations, we evaluate the moments using (11) and the proposed method. We compare the obtained moments with the empirical ones evaluated over $10^{6}$ realizations. The results are summarized in Table [1 As a first observation, for config ${ }_{1}$, we notice that our method provides very close results to the exact moment calculated using [3] with a relative error in the order of $10^{-4}$ as shown in the last

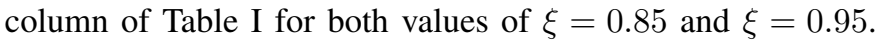
For config ${ }_{2}$, the evaluation of the moments using (1) associated with a higher $q$ becomes completely inaccurate. This clearly demonstrates the efficiency and the accuracy of our method in calculating the positive moments.

\section{Moment-Based Approach for Density APPROXIMATION}

The notion of $M-$ determinate PDFs arose to address the following fundamental question: What should be the condition on the moment sequence $\left\{\mu_{\mathbf{W}}(k)\right\}_{k}$ so that it uniquely determine the $P D F f_{\lambda}(\lambda)$ ? This question has been addressed in $[7,8]$, where sufficient conditions have been specified for the PDF to be fully determined by their moments and hence $M$-determinate.

\footnotetext{
${ }^{1}$ This can be seen by using the fact that $P(X)=$
}

In this vein, we show that the knowledge of all positive moments $\mu_{\mathbf{W}}(k), k=1,2, \cdots$ can be leveraged to approximate the PDF $f_{\lambda}$ as summarized in the theorem below.

Theorem 1. 778 Let $f$ be a distribution defined in the real half-line $(0, \infty)$. If the following conditions are satisfied:

1) The Krein condition: $\int_{0}^{\infty} \frac{-\log f\left(\lambda^{2}\right)}{1+\lambda^{2}} d \lambda=\infty$.

2) The Lin condition: $f$ is differentiable and $\lim _{\lambda \rightarrow \infty} \frac{-\lambda \frac{\partial f(\lambda)}{\partial \lambda}}{f(\lambda)}=\infty$.

Then, $f_{\lambda}$ is M-determinate.

Proposition 1. The PDF in (3) is M-determinate.

Proof: 1) Krein condition: We start by rewriting the PDF in (3) as

$$
f_{\lambda}(\lambda)=\sum_{l=1}^{q} \sum_{k=q-n_{t}+1}^{q} \gamma_{k, l} \lambda^{n_{t}+k-q-1} e^{-\lambda / \beta_{l}},
$$

where $\gamma_{k, l}=\frac{\beta_{l}^{q-n_{t}-1} \boldsymbol{\Psi}_{k, l}^{-1}}{n_{t} \Gamma\left(n_{t}-q+k\right)}$. Then,

$$
\begin{aligned}
f_{\lambda}\left(\lambda^{2}\right) & =\sum_{l=1}^{q} \sum_{k=q-s+1}^{q} \gamma_{k, l} \lambda^{2\left(n_{t}+k-q-1\right)} e^{-\lambda^{2} / \beta_{l}} \\
\leq & e^{-\lambda^{2} / \beta_{q}} \sum_{l=1}^{q} \sum_{k=q-s+1}^{q} \gamma_{k, l} \lambda^{2\left(n_{t}+k-q-1\right)} \\
-\log \left(f_{\lambda}\left(\lambda^{2}\right)\right) & \geq \lambda^{2} / \beta_{q}-\log \left(\sum_{l=1}^{q} \sum_{k=q-n_{t}+1}^{q} \gamma_{k, l} \lambda^{2\left(n_{t}+k-q-1\right)}\right) \\
\frac{-\log \left(f_{\lambda}\left(\lambda^{2}\right)\right)}{1+\lambda^{2}} & \geq \frac{\lambda^{2}}{\beta_{q}\left(1+\lambda^{2}\right)} \\
& -\frac{\log \left(\sum_{l=1}^{q} \sum_{k=q-n_{t}+1}^{q} \gamma_{k, l} \lambda^{2\left(n_{t}+k-q-1\right)}\right)}{1+\lambda^{2}}
\end{aligned}
$$

Integrating the first term of the right-hand side term provides infinity while integrating the second term results in a finite value. Thus, the integral diverges to infinity which fulfills the Krein condition.

2) Lin condition: using the expression in (12), we have

$$
\begin{aligned}
\frac{\partial f_{\lambda}(\lambda)}{\partial \lambda} & =\sum_{l=1}^{q} \sum_{k=q-n_{t}+1}^{q} \gamma_{k, l}\left[\left(n_{t}+k-q-1\right) \lambda^{n_{t}+k-q-2} e^{-\lambda / \beta_{l}}\right. \\
& \left.-1 / \beta_{l} \lambda^{n_{t}+k-q-1} e^{-\lambda / \beta_{l}}\right]
\end{aligned}
$$

$$
\begin{aligned}
& -\lambda \frac{\partial f_{\lambda}(\lambda)}{\partial \lambda}=\sum_{l=1}^{q} \sum_{k=q-n_{t}+1}^{q} \gamma_{k, l} e^{-\lambda / \beta_{l}} \lambda^{n_{t}+k-q-1} \\
& \times\left[-\left(n_{t}+k-q-1\right)+\lambda / \beta_{l}\right] \geq-\left(n_{t}-1\right) f_{\lambda}(\lambda)+\frac{\lambda}{\beta_{q}} f_{\lambda}(\lambda) .
\end{aligned}
$$

Thus, $\frac{-\lambda \frac{\partial f_{\lambda}(\lambda)}{\partial \lambda}}{f_{\lambda}(\lambda)} \geq-\left(n_{t}-1\right)+\frac{\lambda}{\beta_{q}} \stackrel{\lambda \rightarrow \infty}{\longrightarrow} \infty$. 
With this proof, an approximation of the marginal density, involving laguerre polynomials, can be derived as [9]

$$
f_{\lambda}(\lambda)=\frac{\lambda^{\nu} e^{-\lambda / c}}{c^{\nu+1}} \sum_{i=0}^{\infty} \delta_{i} \mathcal{L}_{i}(\nu, \lambda / c),
$$

where $c=\frac{\mu_{\mathbf{W}}(2)-\mu_{\mathbf{W}}(1)^{2}}{\mu_{\mathbf{W}}(1)}, \nu=\frac{\mu_{\mathbf{W}}(1)}{c}-1$,

$$
\mathcal{L}_{i}(\nu, \lambda)=\sum_{k=0}^{i}(-1)^{k} \frac{\Gamma(\nu+i+1) \lambda^{i-k}}{k !(i-k) ! \Gamma(\nu+i-k+1)},
$$

is the Laguerre polynomial of order $i$ in $\lambda$ and parameter $\nu$ and $\delta_{i}=\sum_{k=0}^{i} \frac{(-1)^{k}}{c^{i-k}} \frac{i !}{k !(i-k) ! \Gamma(\nu+i-k+1)} \mu_{\mathbf{W}}(i-k)$. Truncating the series in 18 at order $K$ yields the following approximations for the marginal PDF and CDF

$$
\begin{aligned}
f_{\lambda, K}(\lambda) & =\frac{\lambda^{\nu} e^{-\lambda / c}}{c^{\nu+1}} \sum_{i=0}^{K} \delta_{i} \mathcal{L}_{i}(\nu, \lambda / c) . \\
F_{\lambda, K}(\lambda) & =\sum_{i=0}^{K} \delta_{i} \sum_{k=0}^{i}(-1)^{k} \frac{\gamma(i+\nu-k+1, \lambda / c)}{k !(i-k) !} .
\end{aligned}
$$

\section{SElected numerical Results}

In this section, we investigate the accuracy of the proposed PDF and CDF moment-based approach approximation. To this end, we compare them with their empirical counterparts and those evaluated using the results in [3].

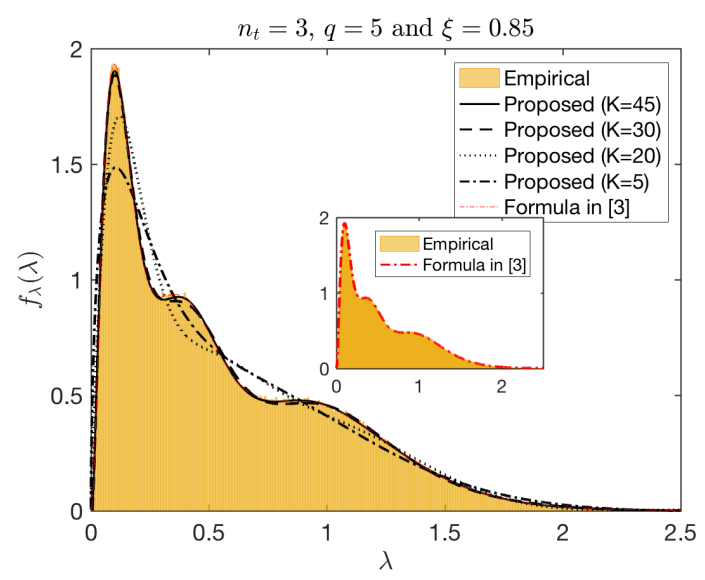

Figure 1: PDF of an unordered eigenvalue of $\mathbf{W}$ with $n_{t}=3$, $q=5$ and $\xi=0.85$.

In Figure 11, we assume that $\boldsymbol{\Lambda}$ follows the same model as in (11) with $\xi=0.85, n_{t}=3$ and $q=5$. We compare the accuracy of our approach with the corresponding empirical density and the formula provided in [3]. It can be noticed that our approximation becomes more accurate by increasing the truncation order $K$. As evidenced from Figures 11 and 2 , a good approximation can be achieved starting from $K=30$ for both PDF and CDF. In Figures 3 and 4 , we increase the value of $q$ to $q=20$. In this case, the formula provided in [3] presents severe numerical instability and thus could not be plotted in this case. On the other hand, our moment-based approach achieves a very good approximation starting from $K=30$. For $K=45$, we can see that we have a perfect match with the empirical PDF and CDF.

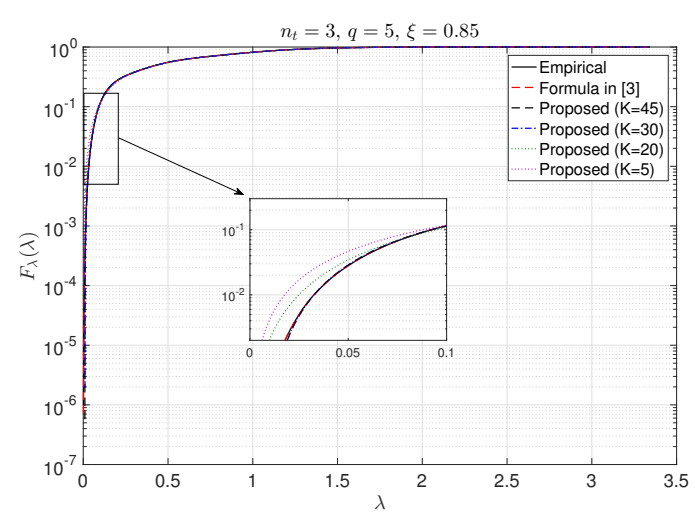

Figure 2: CDF of an unordered eigenvalue of $\mathbf{W}$ with $n_{t}=3$, $q=5$ and $\xi=0.85$.

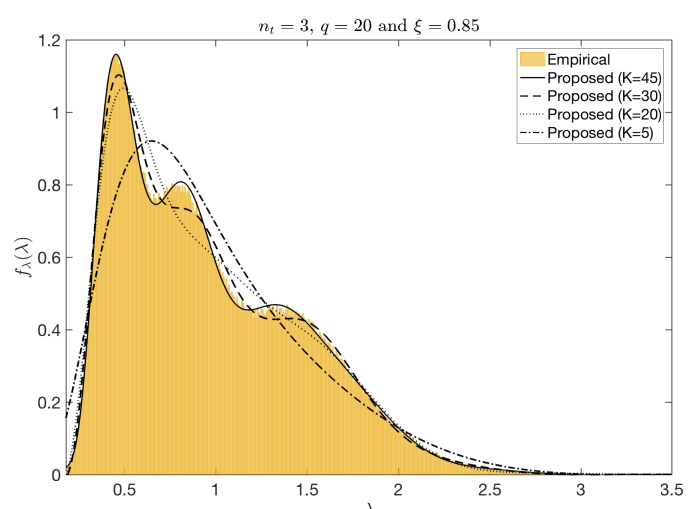

Figure 3: PDF of an unordered eigenvalue of $\mathbf{W}$ with $n_{t}=3$, $q=20$ and $\xi=0.85$. The plot for the exact formula provided in [3] is omitted due to singularity issues.

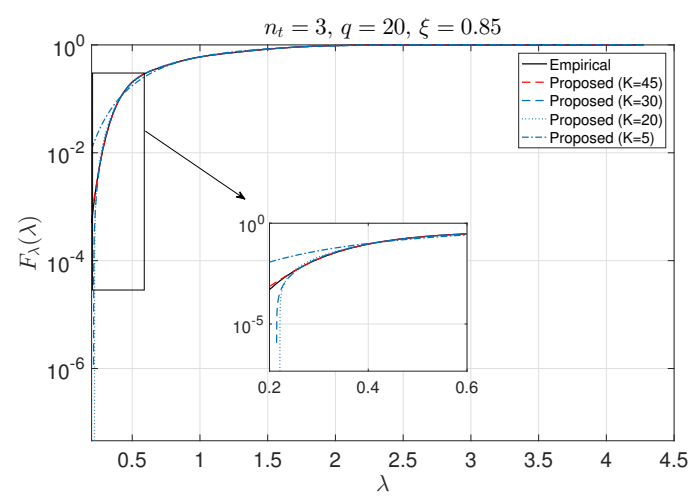

Figure 4: $\mathrm{CDF}$ of an unordered eigenvalue of $\mathbf{W}$ with $n_{t}=3$, $q=20$ and $\xi=0.85$. The plot for the exact formula provided in [3] is omitted due to singularity issues.

\section{CONClusion}

In this paper, we propose a numerically stable method that efficiently compute the positive moments of one-side correlated Gram matrices. From a practical standpoint, these moments can be used to approximate the marginal distribution and CDF of the eigenvalues of Gram random matrices and thus constitute an efficient alternative to conventional methods which become highly inaccurate in high dimensional settings. 


\section{REFERENCES}

[1] K. Elkhalil, A. Kammoun, T. Y. Al Naffouri, and M.-S. Alouini, "Analytical Derivation of The Inverse Moments of One-Sided Correlated Gram Matrices with Applications," IEEE Trans. on Signal Processing, vol. 64, no. 10, pp. 2624-2635, 2016.

[2] G. Alfano, A. M. Tulino, A. Lozano, and S. Verdu, "Capacity of MIMO Channels with One-sided Correlation," ISSSTA, August 2004.

[3] S. Jin, M. R. McKay, C. Zhong, and K. K. Wong, "Ergodic Capacity Analysis of Amplify-and-Forward MIMO Dual-Hop Systems," IEEE Transactions on Information Theory, vol. 56, no. 5, pp. 2204-2224, May 2010.

[4] Milutin Pajovic, "The Development and Application of Random Matrix Theory in Adaptive Signal Processing in the Sample Deficient Regime," Ph.D. dissertation, Massachusetts Institute of Technology, 2014.

[5] A. Zanella, M. Chiani, and M. Z. Win, "On the Marginal Distribution of the Eigenvalues of Wishart Matrices," IEEE Transactions on Communications, vol. 57, no. 4, pp. 1050-1060, April 2009.

[6] R. Seroul, "Newton-Girard Formulas," 10.12 in Programming for Mathematicians. Berlin: Springer-Verlag, pp. 278-279, 2000.

[7] J. Stoyanov, "Krein Condition in Probabilistic Moment Problems," International Statistical Institute (ISI) and the Bernoulli Society for Mathematical Statistics and Probability, vol. 6, no. 5, pp. 939-949, October 2000.

[8] C. R. Rao, Linear Statistical Inference and its Applications. John Wiley \& Sons, 1973.

[9] S. B. Provost, "Moment-Based Density Approximants," The Mathematica Journal, vol. 9, pp. 728-756, 2005. 